\title{
DIAGNÓSTICO E TRATAMENTO DAS PRINCIPAIS SÍNDROMES VESTIBULARES
}

\author{
Aline Mizuta Kozoroski Kanashiro', Cristiana Borges Pereira², \\ Antonio Carlos de Paiva Melo ${ }^{3}$, Milberto Scaff
}

\begin{abstract}
RESUMO - Os objetivos deste estudo foram identificar as síndromes vestibulares mais comuns nos ambulatórios de vertigem, suas características clínicas e semiológicas, e observar a resposta ao tratamento específico. Foram estudados retrospectivamente 515 pacientes atendidos em ambulatórios de duas instituições e avaliados aspectos da anamnese, exame físico e a resposta ao tratamento. As síndromes mais freqüentes foram: vertigem de posicionamento paroxística benigna (VPPB) $(28,5 \%)$, vertigem postural fóbica $(11,5 \%)$, vertigem central $(10,1 \%)$, neurite vestibular $(9,7 \%)$, doença de Menière $(8,5 \%)$, enxaqueca $(6,4 \%)$. Houve boa resposta ao tratamento nos pacientes com enxaqueca $(78,8 \%)$, VPPB $(64 \%)$, neurite vestibular $(62 \%)$, doença de Menière $(54,5 \%)$ e paroxismia vestibular $(54,5 \%)$, enquanto pacientes com nistagmo para baixo e vestibulopatia bilateral não tiveram resposta satisfatória ( $52,6 \%$ e 42,8\% respectivamente). As síndromes vestibulares foram diagnosticadas através da anamnese e exame físico com testes clínicos específicos para avaliação da função vestibular. A identificação destas síndromes permitiu o tratamento adequado levando a uma boa evolução.
\end{abstract}

PALAVRAS-CHAVE: vertigem, etiologia, diagnóstico, tratamento.

\section{Diagnosis and treatment of the most frequent vestibular syndromes}

ABSTRACT - The aims of this study were to identify the most common vestibular syndromes in a dizziness unit, and to observe their clinical aspects and response to treatment. Five hundred and fifteen patients were studied retrospectively in two institutions. Aspects of anamnesis, physical examination and the response to treatment were evaluated. The most frequent syndromes were: benign paroxysmal positioning vertigo (VPPB) (28.5\%), phobic postural vertigo (11.5\%), central vertigo (10.1\%), vestibular neuritis $(9.7 \%)$, Menière disease $(8.5 \%)$, and migraine $(6.4 \%)$. A good response to treatment was observed in most patients with migraine $(78.8 \%)$, VPPB (64\%), vestibular neuritis (62\%), Menière disease $(54.5 \%)$ and vestibular paroxis$\mathrm{mia}(54.5 \%)$. On the other hand, patients with downbeat nystagmus and bilateral vestibulopathy had poor response ( $52.6 \%$ and $42.8 \%$, respectively). The diagnosis of these most frequent vestibular syndromes were established through anamnesis and physical examination (with specific clinical tests for evaluation of the vestibular function). The correct diagnosis and adequate treatment are important since these syndromes may have a good prognosis.

KEY WORDS: vertigo, etiology, diagnosis, treatment.

Vertigem e tontura são sintomas comuns que podem estar envolvidos no comprometimento de múltiplos sistemas e em diferentes síndromes. Muitos dos pacientes com estas queixas são encaminhados para a especialidade de otorrinolaringologia, mas uma parte destes pacientes é encaminhada ao neurologista. No estudo de Ferri-de-Barros e Nitrini ${ }^{1}$, foi encontrada freqüência de $2,77 \%$ pacientes com hipótese diagnóstica de síndrome vestibular encaminhados ao ambulatório de neurologia, mas freqüências de 10 a $20 \%$ também foram relatadas ${ }^{2}$.

Entre as patologias vestibulares deve-se determi- nar se há um comprometimento das estruturas vestibulares centrais ou periféricas, a partir da história clínica e exame físico, pois o tratamento e evolução são muito diferentes ${ }^{2}$.

Os objetivos deste estudo foram: (1) identificar as síndromes vestibulares mais comuns encontradas nos ambulatórios de vertigem, suas principais características clínicas e semiológicas e (2) observar a resposta ao tratamento específico.

\section{MÉTODO}

O estudo foi realizado por meio da análise retrospecti-

Hospital do Servidor Público Estadual de São Paulo (HSPE) e Hospital das Clínicas da Faculdade de Medicina da Universidade de São Paulo (HC-FMUSP) São Paulo, SP, Brasil: 'Médica estagiária do Ambulatório de Vertigem do Serviço de Neurologia do HSPE; ${ }^{2}$ Doutora do Departamento de Neurologia da FMUSP, responsável pelo ambulatório de Distúrbios Vestibulares do HC-FMUSP e Médica assistente do HSPE e responsável pelo ambulatório de Vertigem do HSPE; ${ }^{3}$ Diretor do Serviço de Neurologia do HSPE; ${ }^{4}$ ProfessorTitular do Departamento de Neurologia da FMUSP.

Recebido 26 Maio 2004, recebido na forma final 18 Agosto 2004. Aceito 7 Outubro 2004.

Dra.Aline Mizuta Kozoroski Kanashiro - Rua Martiniano de Carvalho 629/84 -01321-001 São Paulo SP-Brasil. E-mail: kozoroski@terra.com.br 
va dos prontuários dos pacientes atendidos no Ambulatório de Vertigem, na Divisão de Clínica Neurológica do Hospital das Clínicas da Faculdade de Medicina da Universidade de São Paulo, no período de novembro de 1999 a dezembro de 2003; e no Ambulatório de Vertigem do Serviço de Neurologia do Hospital do Servidor Público Estadual de São Paulo, durante o período de setembro de 2002 a março de 2004.

Foram avaliados os prontuários de 515 pacientes encaminhados por médicos do Serviço de Neurologia (Pronto Socorro e Ambulatório), médicos de outras clínicas, principalmente devido a queixas de tonturas. Esses pacientes foram submetidos a anamnese dirigida ao sistema vestibular, exame neurológico, com atenção especial para o exame de equilíbrio estático e dinâmico, avaliação de seguimento, sacadas, pesquisa das diferentes formas de nistagmo (espontâneo, de provocação, posicional, optocinético) e reflexo vestíbulo-ocular. Exames complementares (laboratoriais e radiológicos) foram solicitados para confirmação diagnóstica e para avaliar a função do labirinto, mas não foram analisados neste estudo.

A partir do diagnóstico foi proposto o tratamento específico. A resposta ao tratamento foi avaliada no retorno dos pacientes.

Através das informações colhidas nos ambulatórios foi elaborado um banco de dados contendo: (1) informações sobre o paciente (nome, registro, idade, gênero); (2) queixa principal, tipo de vertigem e sintomas associados; (3) exame físico; (4) diagnóstico; (5) tratamento; (6) evolução.

\section{RESULTADOS}

Do total de pacientes avaliados nos ambulatórios, as patologias mais freqüentes, incluindo as vestibulares e não vestibulares, estão no Gráfico 1. E ntre aqueles com diagnóstico de síndrome vestibular periférica estão incluídos as seguintes doenças: vertigem de posicionamento paroxística benigna (VP$\mathrm{PB})$, doença de Menière, neurite vestibular, paroxismia vestibular, vestibulopatia bilateral e outras doenças menos comuns (colesteatoma, mastoidite, sarcoidose, uso de antibiótico ototóxico, neurinoma do acústico).

O Gráfico 2 mostra a freqüência das doenças vestibulares periféricas, centrais, vertigem postural fóbica, e enxaqueca e exclui os casos de tontura de causa não vestibular. No grupo identificado como "outros" foram agrupados os diagnósticos de paroxismia vestibular, vestibulopatia bilateral, colesteatoma, mastoidite, sarcoidose, uso de antibiótico ototóxico, neurinoma do acústico. Dentre as vertigens centrais estão incluídos os pacientes com nistagmo vertical, downbeat (para baixo) e upbeat (para cima), ambas caracterizadas como síndrome vestibular central no plano vertical ou "pitch", e os pacientes com nistagmo rotatório, desvio skew (de- salinhamento vertical), caracterizado por síndrome vestibular central no plano frontal ou "roll".

Na Tabela 1 estão distribuídas as doenças vestibulares conforme sua freqüência assim como média de idade e gênero dos pacientes. A Tabela 2 mostra a distribuição das diferentes síndromes vestibulares encontradas nos ambulatórios de vertigem comparada à freqüência relatada na literatura. As características clínicas e semiológicas, e o tratamento das

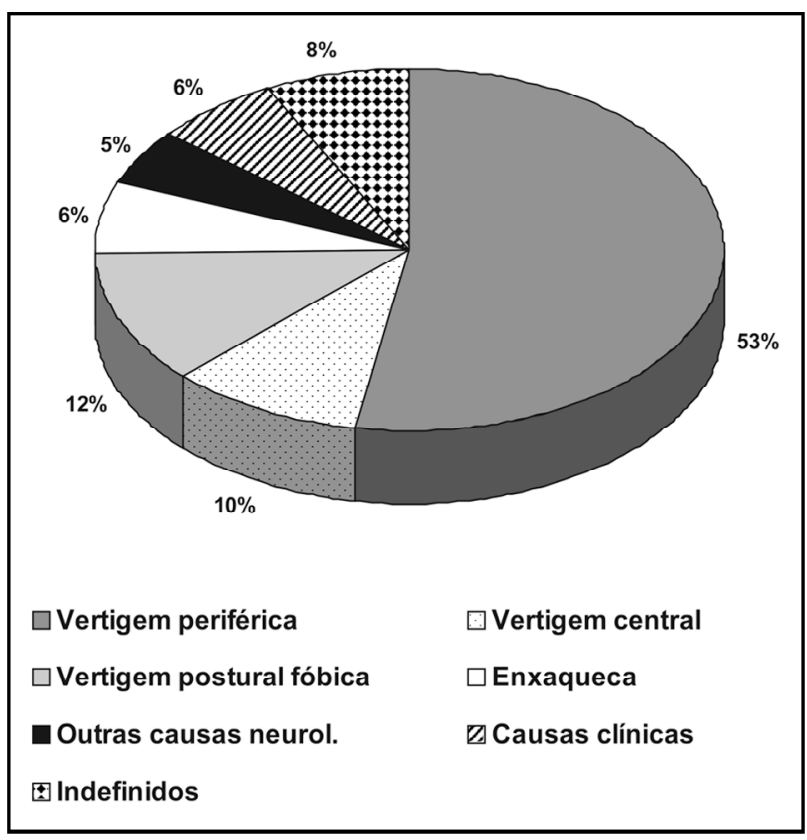

Gráfico 1. Distribuição de freqüências das síndromes vestibulares e não vestibulares no ambulatório de vertigem.

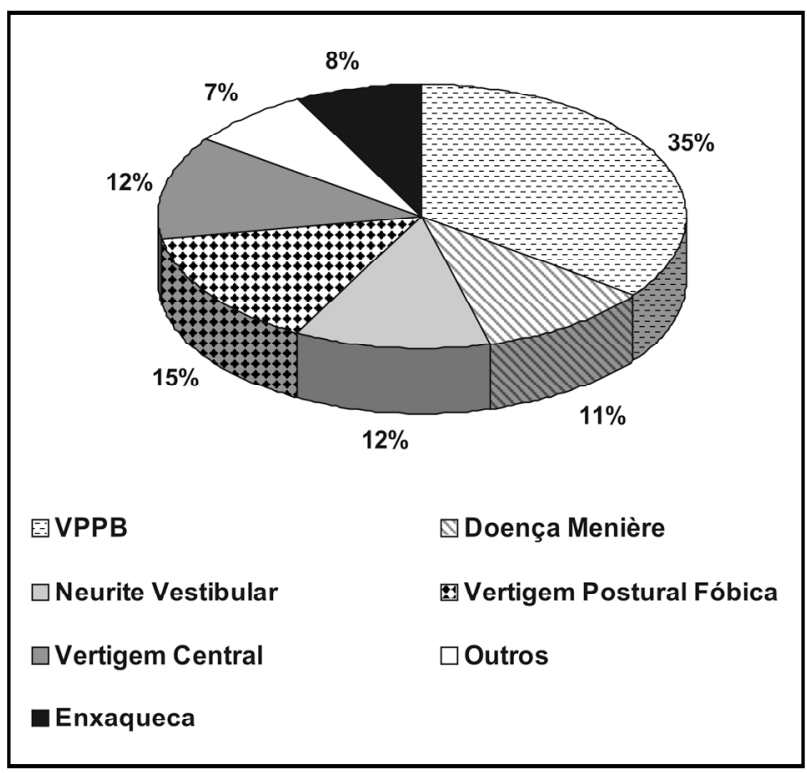

Gráfico 2. Distribuição de freqüências das doenças vestibulares periféricas, centrais, vertigem postural fóbica, e enxaqueca. 
Quadro 1. Características clínicas e semiológicas e o tratamento das principais síndromes vestibulares periféricas, centrais, vertigem postural fóbica e enxaqueca.

\begin{tabular}{|c|c|c|c|}
\hline Diagnóstico & Características da anamnese & Características do exame físico & Tratamento \\
\hline VPPB & $\begin{array}{l}\text { Vertigem rotatória de curta duração } \\
\text { desencadeada por movimentos rápidos da } \\
\text { cabeça }^{5}\end{array}$ & $\begin{array}{l}\text { Nistagmo de posicionamento vertical para } \\
\text { cima, rotatório para o lado comprometido }\end{array}$ & $\begin{array}{l}\text { Manobra de Epley }{ }^{6} \text {, manobra } \\
\text { de Semont }{ }^{7} \text {, manobra de } \\
\text { Brandt-Daroff } 8\end{array}$ \\
\hline $\begin{array}{l}\text { Doença de } \\
\text { Menière }\end{array}$ & $\begin{array}{l}\text { Tríade de ataques recorrentes de vertigem, } \\
\text { déficit auditivo flutuante, zumbidos, } \\
\text { pressão no ouvido do lado comprometido9 }\end{array}$ & $\begin{array}{l}\text { Durante as crises: nistagmo horizonto- } \\
\text { rotatório para o lado lesado ou para o lado } \\
\text { são, desequilíbrio, hipoacusia }{ }^{9}\end{array}$ & Betaistina, diurético ${ }^{9}$ \\
\hline $\begin{array}{l}\text { Neurite } \\
\text { vestibular }\end{array}$ & $\begin{array}{l}\text { Vertigem rotatória aguda de duração } \\
\text { prolongada, desequilíbrio, tendência a } \\
\text { queda para um dos lados, náuseas, vômitos. } \\
\text { Ausência de sintomas auditivos }\end{array}$ & $\begin{array}{l}\text { Tendência de queda para o lado lesado, } \\
\text { nistagmo horizonto-rotatório para o lado } \\
\text { são, reflexo vestíbulo-ocular patológico no } \\
\text { lado da lesão }{ }^{10}\end{array}$ & Reabilitação vestibular \\
\hline $\begin{array}{l}\text { Paroxismia } \\
\text { vestibular }\end{array}$ & $\begin{array}{l}\text { Ataques curtos e freqüentes de vertigem } \\
\text { rotatória desencadeados por movimentos da } \\
\text { cabeça, podem ser associados a tinitus } \\
\text { ou hipoacusia }\end{array}$ & $\begin{array}{l}\text { Nistagmo de provocação para o lado bom. } \\
\text { Pode ter associado paralisia facial, espasmo } \\
\text { facial, hipoacusia, ispsilaterais }{ }^{3}\end{array}$ & Carbamazepina, fenitoína ${ }^{3}$ \\
\hline $\begin{array}{l}\text { Vestibulopatia } \\
\text { bilateral }\end{array}$ & Desequilíbrio, oscilopsia. & $\begin{array}{l}\text { Reflexo vestíbulo-ocular } \\
\text { patológico bilateral }\end{array}$ & $\begin{array}{l}\text { Reabilitação vestibular, } \\
\text { tratamento da causa }{ }^{3}\end{array}$ \\
\hline $\begin{array}{l}\text { Outras lesões } \\
\text { periféricas }\end{array}$ & $\begin{array}{l}\text { Além de sintomas e sinais de vertigem } \\
\text { periférica, sintomas auditivos como } \\
\text { hipoacusia, dor no ouvido, dor em } \\
\text { região da mastóide. }\end{array}$ & $\begin{array}{l}\text { Hipoacusia, reflexo vestíbulo-ocular } \\
\text { patológico no lado da lesão }\end{array}$ & Tratamento da causa ${ }^{3}$ \\
\hline $\begin{array}{l}\text { Vertigem } \\
\text { postural } \\
\text { fóbica }\end{array}$ & $\begin{array}{l}\text { Vertigem oscilatória ou tontura } \\
\text { desencadeadas em locais movimentados e } \\
\text { cheios de pessoas, com multidões, associada a medo } \\
\text { de sair de casa, medo de cair, ansiedade }{ }^{11}\end{array}$ & Exame normal & $\begin{array}{l}\text { Antidepressivo (tricíclico ou } \\
\text { inibidor da recaptação de } \\
\text { dessensibilização'11 }\end{array}$ \\
\hline $\begin{array}{l}\text { Vertigem } \\
\text { central }\end{array}$ & $\begin{array}{l}\text { Sintomas de uma síndrome vestibular } \\
\text { (vertigem, náuseas, vômitos, desequilíbrio, } \\
\text { nistagmo) associados a sintomas de } \\
\text { comprometimento do } \mathrm{SNC}^{3}\end{array}$ & $\begin{array}{l}\text { Diplopia, oftalmoparesia internuclear, } \\
\text { desvio skew, nistagmo upbeat (vertical para } \\
\text { cima), ataxia cerebelar, outros sintomas de } \\
\text { comprometimento de tronco encefálico }{ }^{3}\end{array}$ & $\begin{array}{l}\text { Tratamento da causa (AVC, } \\
\text { esclerose múltipla, infecções } \\
\text { do SNC, outros) }\end{array}$ \\
\hline $\begin{array}{l}\text { Nistagmo } \\
\text { downbeat } \\
\text { e upbeat }\end{array}$ & $\begin{array}{l}\text { Vertigem oscilatória, náuseas, vômitos, } \\
\text { desequilíbrio, nistagmo, e sintomas de } \\
\text { comprometimento do tronco encefálico }\end{array}$ & $\begin{array}{l}\text { Nistagmo downbeat: vertical para baixo. } \\
\text { Nistagmo upbeat: vertical para cima }\end{array}$ & $\begin{array}{l}\text { Benzodiazepínico, lioresal, } \\
\text { tratamento da causa }{ }^{3}\end{array}$ \\
\hline Enxaqueca & Critérios International Headache Society (IHS) ${ }^{12}$ & $\begin{array}{l}\text { Exame normal ou com pequenas alterações } \\
\text { da motricidade ocular extrínseca } \\
\text { (seguimento e sacadas) }^{3}\end{array}$ & $\begin{array}{l}\text { Betabloqueador, } \\
\text { antidepressivo }^{3}\end{array}$ \\
\hline
\end{tabular}

Tabela 1. Distribuição de frequências das síndromes vestibulares, da média de idade e de gênero dos pacientes.

\begin{tabular}{lcccc}
\hline Diagnóstico & $\mathrm{n}(\%)$ & Média de & \multicolumn{2}{c}{ Gênero } \\
\cline { 4 - 5 } & & idade & Fem & Masc \\
\hline VPPB & $147(35,2)$ & 65,3 & 108 & 39 \\
Doença de Menière & $44(10,5)$ & 54,4 & 36 & 8 \\
Neurite vestibular & $50(12)$ & 52,9 & 26 & 24 \\
Vertigem postural fóbica & $61(14,6)$ & 52,3 & 47 & 14 \\
Vertigem central no plano frontal ou roll & $31(7,4)$ & 56,2 & 18 & 13 \\
Paroxismia vestibular & $11(2,6)$ & 62,5 & 9 & 2 \\
Enxaqueca & $33(7,9)$ & 50,7 & 30 & 3 \\
Nistagmo para baixo (downbeat) & $21(5,0)$ & 61,1 & 13 & 8 \\
e para cima (upbeat) - vertigem & & & & \\
central no plano vertical ou pitch & & & 5 & 2 \\
Vestibulopatia bilateral & $7(1,7)$ & 57,6 & 10 & 3 \\
Outras lesões periféricas & $13(3,1)$ & 56 & 302 & 116 \\
Total & $418(100)$ & 57,8 & & \\
\hline
\end{tabular}

principais doenças vestibulares periféricas, centrais e vertigem postural fóbica estão no Quadro 1. Na Tabela 3 é mostrada a evolução verificada com o acompanhamento dos pacientes.

\section{DISCUSSÃO}

Vertigem e tontura são consideradas sinônimos no dicionário Aurélio, no entanto, do ponto de vista médico estes termos podem ser utilizados com o seguinte significado: (1) "vertigem rotatória": define a sensação de que tudo roda ao redor de si mesmo ou que se está girando, freqüentemente associada a náuseas, vômitos e desequilíbrio. Esta queixa está presente quando há acometimento vestibular unilateral (periférico ou central); (2) "vertigem oscilatória": refere-se à sensação de balanços ou de per- 
Tabela 2. Distribuição de freqüências das síndromes vestibulares encontradas nos ambulatórios de vertigem comparada às freqüências relatadas na literatura.

\begin{tabular}{lcc}
\hline Diagnóstico & $\begin{array}{c}\text { Ambulatório de vertigem } \\
\mathrm{n}(\%)\end{array}$ & $\begin{array}{c}\text { Literatura } \\
\mathrm{n}(\%)\end{array}$ \\
\hline VPPB & $147(28,5)$ & $395(19,6)$ \\
Vertigem posturalfóbica & $61(11,5)$ & $320(15,9)$ \\
Vertigem central & $52(10,1)$ & $292(14,5)$ \\
Neurite vestibular & $50(9,7)$ & $134(6,7)$ \\
Doença de Menière & $44(8,5)$ & $151(7,5)$ \\
Enxaqueca & $33(6,4)$ & $159(7,9)$ \\
Outras lesões periféricas & $13(2,6)$ & $5(0,3)$ \\
Paroxismia vestibular & $11(2,1)$ & $41(2,0)$ \\
Vestibulopatia bilateral & $7(1,4)$ & $52(2,6)$ \\
Vertigem psicogênica & $0(0)$ & $49(2,4)$ \\
Indefinidos & $39(7,6)$ & $96(4,8)$ \\
Causas clínicas & $33(6,4)$ & $0(0)$ \\
Outros & $25(4,9)$ & $316(15,8)$ \\
\hline
\end{tabular}

Tabela 3. Dados da evolução clínica após o tratamento específico das síndromes vestibulares.

\begin{tabular}{lcccc}
\hline Diagnóstico & \multicolumn{3}{c}{ Evolução - n (\%) } & $n$ \\
\cline { 2 - 4 } & Melhora & Sem melhora & Indefinido \\
\hline VPPB & $94(64)$ & $14(9,5)$ & $39(26,5)$ & 147 \\
Doença de Menière & $24(54,5)$ & $2(4,5)$ & $18(41)$ & 44 \\
Neurite vestibular & $31(62)$ & $4(8)$ & $15(30)$ & 50 \\
Vertigem postural fóbica & $31(50,8)$ & $12(19,7)$ & $18(29,5)$ & 61 \\
Vertigem central no plano frontal ou roll & $11(35,5)$ & $11(35,5)$ & $9(29)$ & 31 \\
Paroxismia vestibular & $6(54,5)$ & $0(0)$ & $5(45,5)$ & 11 \\
Enxaqueca & $26(78,8)$ & $2(6,1)$ & $5(15,1)$ & 33 \\
Nistagmo para baixo (downbeat) & $1(4,8)$ & $11(52,4)$ & $9(42,8)$ & 21 \\
e para cima (upbeat) - vertigem central & & & & 7 \\
no plano vertical ou pitch & $1(14,4)$ & $3(42,8)$ & $3(42,8)$ & 13 \\
Vestibulopatia bilateral & $7(53,8)$ & $2(15,4)$ & $4(30,8)$ & \\
Outras lesões periféricas & & &
\end{tabular}

da de equilíbrio, raramente associada a náuseas ou vômitos, e pode ser devida a distúrbios vestibulares ou não vestibulares; (3) "tontura": é considerada como mal-estar, escurecimento visual, fraqueza, sintomas muitas vezes vagos sem ter a sensação de rotação ou desequilíbrio, e tem causa não neurológica, como exemplo: hipotensão postural, ataque vasovagal, arritmia cardíaca, hipoglicemia, e outras causas ${ }^{3}$.

Durante a realização da anamnese foi importante a diferenciação entre os sintomas vertigem e tontura, que correspondem ao comprometimento de estruturas distintas. Os termos vertigem rotatória, vertigem oscilatória e tontura foram utilizados para classificar as queixas dos pacientes, o que facilitou o estabelecimento de diagnósticos diferenciais (Tabela 1).
No Gráfico 1 estão distribuídos todos os pacientes atendidos nos ambulatórios de vertigem, com doenças vestibulares e não vestibulares (outras causas neurológicas, causas clínicas e indefinidos). $\mathrm{O}$ Gráfico 2 mostra a distribuição de freqüências somente dos distúrbios do sistema vestibular. Em ambos os gráficos, a vertigem postural fóbica e enxaqueca foram analisadas em separado por não se enquadrarem nas doenças vestibulares periféricas ou centrais.

A distribuição das doenças encontradas nos ambulatórios apresentou pequenas diferenças comparada à relatada na literatura ${ }^{2}$ (Tabela 2 ). Houve grande porcentagem de pacientes com VPPB, acima dos valores encontrados por outros autores. A explicação para essa distribuição é um provável viés 
devido à realização de um estudo sobre VPPB no mesmo período, direcionando os pacientes com queixas sugestivas desta síndrome aos ambulatórios. As pequenas diferenças na freqüência de pacientes com neurite vestibular e enxaqueca são pouco relevantes se considerado o fato dos estudos terem sido realizados em populações diferentes.

Os diagnósticos de vertigem central no plano "roll" e de nistagmos downbeat e upbeat (vertigem central no plano "pitch") foram separados porque são devidos a lesões distintas (unilateral no primeiro caso e bilateral no último) e tiveram tratamentos diferentes ${ }^{4}$.

A freqüência de causas clínicas (Tabela 1) é difícil de ser avaliada e comparada pelas seguintes razões: (1) muitos pacientes provavelmente foram encaminhados por médicos clínicos, e as causas clínicas de tontura foram identificadas e tratadas sem necessidade de encaminhamento ao ambulatório de vertigem; (2) o relato da literatura não considerou este diagnóstico².

A distribuição entre os gêneros variou conforme cada diagnóstico e o resultado está na Tabela 1 a média de idade foi 57,8 , que pode ser explicada pelo fato destas patologias vestibulares atingirem indivíduos em faixa etária mais alta, variando pouco em cada doença.

O tratamento foi específico para cada patologia $^{5-12}$ (Quadro 1), e a resposta ao tratamento foi avaliada com o acompanhamento dos pacientes. A evolução foi classificada em: (1) com melhora, ou seja, desaparecimento do nistagmo (p. ex: VPPB, neurite vestibular), diminuição do nistagmo (p. ex: nistagmo downbeat, upbeat, paroxismia), e diminuição da freqüência das crises (p. ex: doença de Menière, enxaqueca, vertigem postural fóbica); (2) sem melhora, quando houve persistência do sintoma e/ou alterações no exame físico; (3) indefinida, devido à perda de seguimento. $O$ tratamento das doenças vestibulares periféricas incluiu a recuperação da função vestibular (p. ex: neurite vestibular), o controle e/ ou atuação na fisiopatologia da síndrome ( $p$. ex: VPPB, doença de Menière, paroxismia vestibular, vertigem postural fóbica). Em relação aos pacientes com vertigem central o tratamen- to proposto foi aquele adequado à doença de base. Os dados da Tabela 3 mostram que a maioria dos pacientes teve boa evolução com o tratamento, significando que há bom prognóstico quando as doenças vestibulares são tratadas adequadamente.

Houve, porém, duas exceções em relação ao tratamento, dentre elas o nistagmo downbeat e a vestibulopatia bilateral, que tiveram pior prognóstico. No nistagmo downbeat há comprometimento bilateral de estruturas centrais com difícil compensação e, portanto, pouca resposta ao tratamento específico. Na vestibulopatia bilateral não ocorre compensação e/ou substituição satisfatória do sistema vestibular periférico comprometido em ambos os lados.

Através deste estudo retrospectivo, realizado com 515 pacientes atendidos em ambulatórios de vertigem pôde-se concluir que: (1) A partir da anamnese e exame físico dirigidos ao sistema vestibular foi possível estabelecer o diagnóstico das síndromes vestibulares e não vestibulares. (2) As síndromes mais freqüentes foram: VPPB, vertigem postural fóbica, vertigem central, neurite vestibular, doença de Menière, enxaqueca. (3) O tratamento específico levou a uma boa evolução na maioria dos pacientes.

\section{REFERÊNCIAS}

1. Ferri-de-Barros JE, Nitrini R. Que pacientes atende um neurologista? Alicerce de um currículo em neurologia. Arq Neuropsiquiatr 1996;54:637-644.

2. Brandt T. Vertigo, its multisensory syndromes, $2^{\text {nd }}$ Ed. London: Springer Verlag, 1999.

3. Velasco IT, Scalabrini A Neto, Karan JA, Cruz LM Neto, Caramez MPR, Gattaz MD. Propedêutica na emergência. São Paulo:Atheneu, 2003.

4. Brandt $t$, Dieterich M. Central vestibular syndromes in roll, pitch and yaw planes: topographic diagnosis of brainstem disorders. Neuroophthalmology 1995;15:291-303.

5. Furman JM, Cass SP. Benign paroxysmal positional vertigo. N Engl J Med 1999;341:1590-1596.

6. Epley JM. The canalith repositioning procedure for treatment of benign paroxysmal positioning vertigo. Otolaryngol Head Neck Surg 1992; 107:299-304.

7. Semont A, Freyss E, VitteP. Curing the BBPV with a liberatory maneuver. Adv Oto-Rhino-Laryngol 1988;42:290-293.

8. Brandt T, Daro ffRB. Physical therapy for benign paroxysmal positional vertigo. Arch Otolaryngol 1980;106:484-485.

9. Hamann KF, Arnold W. Menière's disease. Adv otorhino-laryngol 1999;55:137-168.

10. Baloh RW. Vestibular neuritis. N Engl J Med 2003;348:1027-1032.

11. Brandt T. Phobic postural vertigo. Neurology 1996;46:1515-1519.

12. Headache Classification Committee of the International Headache Society. Classification and diagnostic criteria for headache disorders, cranial neuralgia, and facial pain. Cephalalgia 1988;8(Suppl.7):S1-S96. 